\title{
Regulation of cell cycle transition and induction of apoptosis in HL-60 leukemia cells by the combination of Coriolus versicolor and Ganoderma lucidum
}

\author{
TZE-CHEN HSIEH and JOSEPH M. WU
}

Department of Biochemistry and Molecular Biology, New York Medical College, Valhalla, NY 10595, USA

Received February 6, 2013; Accepted May 8, 2013

DOI: $10.3892 / \mathrm{ijmm} .2013 .1378$

\begin{abstract}
Medicinal mushrooms have served as the mainstay of treatment for a variety of human illnesses in Asian countries, mostly as supplements by cancer patients. Extracts prepared from Trametes versicolor under the trade name of I'm-Yunity exhibit anti-tumorigenic activities, as supported by inhibition of the proliferation and induction of apoptosis in malignant cells. Similar effects have also been observed for the Reishi mushroom Ganoderma lucidum. The two mushrooms exert their medicinal activities primarily through a family of polysaccharo-peptides. Despite the common identity in their bioactive ingredients, whether their combination might elicit an expanded efficacy and mechanism has not been investigated. In the present study, we investigated similarities and differences between extracts prepared from I'm-Yunity and from a formulation denoted I'm-Yunity-Too combining I'm-Yunity and Ganoderma lucidum. By assaying their anti-proliferative and anti-apoptotic effects using human promyelocytic HL-60 cells, we found that the ethanolic extract of I'm-Yunity-Too was more active in inducing cell death compared to I'm-Yunity, based on measured changes in the expression of caspase 3 and Bax. Moreover, ethanolic extracts of I'm-Yunity-Too exhibited more potent activity compared to its aqueous extracts with regard to suppression of the growth and induction of apoptosis, as assayed by the more pronounced downregulation of phosphorylation of $\mathrm{Rb}$ and increased cleavage of poly(ADP-ribose) polymerase (PARP) from its native $112-\mathrm{kDa}$ form to the inactive $89-\mathrm{kDa}$ product. These results suggested that the chemopreventive potential of I'm-Yunity may be enhanced by adding Ganoderma lucidum and that their bioactive ingredients potentially exhibit mechanistic synergism suggesting a more efficacious adjunct in chemotherapy.
\end{abstract}

Correspondence to: Professor Joseph M. Wu, Department of Biochemistry and Molecular Biology, New York Medical College, Valhalla, NY 10595, USA

E-mail: joseph_wu@nymc.edu

Key words: Coriolus versicolor, Ganoderma lucidum, cell cycle control, apoptosis

\section{Introduction}

Cancer remains a significant cause of morbidity and mortality in the United States and worldwide, despite advances made in recent decades on early detection and diagnosis. Increasingly, among practicing clinicians and research professionals, the one-time dominant view of seeking 'magic bullets' for cancer eradication has been supplanted by the objective of cancer prevention and management in order to improve the quality of life of cancer patients.

A reductionist approach to curtail cancer risks, thereby preventing cancer, involve the elimination or reduction, of exposure to sources of carcinogens, together with change in lifestyle and dietary habits. It is therefore notable that, although anti-tumorigenic agents abound in the diet, as revealed by epidemiological/migrant studies reporting an inverse relationship between diet and cancer incidence rate, evidence indicates that diet harbors carcinogens or their precursors. This dual benefit/risk nature of diet with regard to carcinogenesis was clearly demonstrated in a seminal report by Doll and Peto (1) who suggested that $10-70 \%$ of human cancer mortality may be associated with diet. Furthermore, whereas studies by Steinmetz and Potter $(2,3)$ and Willett (4) portend that dietary factors contribute to one third of all cancer deaths, evidence indicates that an estimated $20-50 \%$ of all cancer types are preventable using plant-based diet strategies $(5,6)$, in part attributed to phytochemicals and polyphenols with chemopreventive activities (7-11).

The Reishi mushroom Ganoderma lucidum belongs to the Basidiomycetes class of fungi whose use as an antitumor agent dated back to the Imperial Court of ancient China (12-14). The major bioactive compounds in Ganoderma lucidum are polysaccharides, ganoderic, ganodermic, lucidic acids and their aldehydes and alcohols, and highly oxidized lanostanetype triterpenes (15-17). I'm-Yunity ${ }^{\mathrm{TM}}$ is a proprietary method procured version of the Basidomycetes mushroom comprising a heterogeneous family of polysaccharide-protein complexes isolated from the cultivated mycelia of Trametes (formerly Coriolus) versicolor, which have been used as a major ingredient in traditional Chinese medicinal formulations for the prevention and treatment of chronic diseases including cancer (18-27). The two mushroom products are available as dietary supplements, principally as adjunct immune boosters and prophylactic chemopreventative agents targeting the initiation 
and progression of cancers $(12,20,23,28-40)$. Despite their similar biological activities, differences may exist, both with respect to the nature of the polysaccharides and the spectrum of synthesized and secreted secondary metabolites. Accordingly, it may be postulated that the combination of the two mushrooms elicits distinct as well as overlapping biological and molecular effects, compared to single mushroom extracts. However, this possibility has yet to be investigated. In this study, extracts prepared from I'm-Yunity and from a combined formulation denoted I'm-Yunity-Too ${ }^{\mathrm{TM}}$-containing extracts derived from I'm-Yunity and Ganoderma lucidum were investigated with regard to differences in: i) anti-proliferative activities; ii) cell cycle control and induction of apoptosis; iii) activities between aqueous and ethanolic extracts.

\section{Materials and methods}

Reagents. Fetal calf serum, RPMI-1640, penicillin and streptomycin were purchased from Cellgro, Inc. (Herndon, VA, USA). Any other chemicals and solvents were of analytical grade. Primary antibodies, respectively, against Rb, E2F, NF- $\mathrm{B}$ p p0, NF- $\kappa$ B p65, I $\mathrm{B}$, caspase 3, bcl 2, Bax, actin, and secondary antibodies were purchased from Santa Cruz Biotechnology, Inc. (Santa Cruz, CA, USA). Primary antibodies against phosphorylated Rb Ser780, Ser795, Ser807, Thr821 and Thr826 were obtained from Biosource International, Inc. (Camarillo, CA, USA). Anti-PARP was purchased from Biomol International, L.P. (Plymouth Meeting, PA, USA).

Source of Coriolus versicolor and Ganoderma lucidum. I'm-Yunity and I'm-Yunity-Too capsules containing extracts of Coriolus versicolor, and of Coriolus versicolor and Ganoderma lucidum, respectively, (lot 3BA03020528) were provided by Integrated Chinese Medicine Holdings, Ltd. (Hong Kong, China). The mushroom products were extracted and purified from mycelia of Coriolus versicolor and Ganoderma lucidum according to Good Manufacturing Practice (GMP) standards. Quality control assays validating their authenticity and integrity were performed in government-approved testing centers in Hong Kong.

Preparation of aqueous and ethanolic extracts of I'm-Yunity and I'm-Yunity-Too. To prepare aqueous or ethanolic extracts of I'm-Yunity and I'm-Yunity-Too, the contents of each capsule were suspended in $6 \mathrm{ml}$ of water or $70 \%$ ethanol, intermittently mixed by vortexing for $60 \mathrm{~min}$ at room temperature, and centrifuged to remove insoluble particles. The clear supernatant was sterilized by passing through a $0.22 \mu \mathrm{m}$ filter and stored in aliquots at $4^{\circ} \mathrm{C}$.

Cell culture and growth inhibition assay. Human promyelocytic leukemia cell line (HL-60) was supplied by American Tissue Culture Collection (Manassas, VA, USA). Cells were cultured in RPMI-1640 and seeded at a density of $3 \times 10^{5}$ cells $/ \mathrm{ml}$ for all the experiments, as previously described (24,26,41-43). Extracts of I'm-Yunity and I'm-Yunity-Too were added to the culture media at the final doses specified. Control and mushroom extract-exposed cells were harvested at indicated times after treatment. Cell count was performed using a hemocytometer and viability was determined by trypan blue exclusion. Harvested cells were washed twice with PBS, and pellets were stored at $-80^{\circ} \mathrm{C}$ for subsequent analysis.

Cell cycle analysis. Cell cycle phase distribution was assayed by flow cytometry. Following a 24-, 48- and 72-h treatment of HL-60 cells with aqueous and ethanol extracts of I'm-Yunity or I'm-Yunity-Too $(10 \mu \mathrm{l} / \mathrm{ml})$, the cells were stained with $1.0 \mu \mathrm{g} / \mathrm{ml}$ DAPI containing $100 \mathrm{mM} \mathrm{NaCl}, 2 \mathrm{mM} \mathrm{MgCl}_{2}$ and $0.1 \%$ Triton X-100 (Sigma-Aldrich Corp., St. Louis, MO, USA) at pH 6.8, as previously described (24,26,41-43). The DNA contents were collected and analyzed by a flow cytometer (Ortho Diagnostic, Westwood, MA, USA) and MultiCycle software from Phoenix Flow Systems (San Diego, CA, USA) was used to quantify the percentage of cells in the respective phases $\left(G_{1}, S\right.$ and $\left.G_{2} / M\right)$ of the cell cycle, as previously described (24,26,41-43). Flow cytometry was used to determine cells undergoing apoptosis, as evident by the appearance of the sub- $\mathrm{G}_{1}$ peak (44-46).

Preparation of cell extracts and western blot analysis. For immunoblotting experiments, cells were collected and lysed in ice-cold RIPA buffer, as described in recent studies (47-50). The aliquots of lysates ( $20 \mu \mathrm{g}$ of protein) were boiled with sample buffer for $5 \mathrm{~min}$, and resolved by $10 \%$ SDS-PAGE. The proteins were transferred to a nitrocellulose membrane and blocked with TBST buffer (10 mM Tris, pH 7.5, $100 \mathrm{mM}$ $\mathrm{NaCl}$ and $0.05 \%$ Tween-20) containing 3\% non-fat dried milk overnight at $4{ }^{\circ} \mathrm{C}$. The blots were incubated overnight with various primary antibodies followed by a 1-h incubation with secondary antibodies. The blots were detected with an ECL detection system (LumiGLO Peroxidase Chemiluminescent Substrate kit, KPL Biotechnology, Inc., Gaithersburg, MD, USA) and quantified by densitometry. Actin expression was used for normalization of the sample loading, as previously described (47-50).

\section{Results}

Differential inhibition of cell growth by aqueous and ethanolic extracts of I'm-Yunity and I'm-Yunity-Too. To determine whether aqueous and ethanolic extracts of I'm-Yunity and I'm-Yunity-Too exert comparable anti-proliferative activities, human leukemia HL-60 cells were exposed to a single dose of either extracts for 24-72 h. Growth and cell viability were measured as described in Materials and methods. Results in Fig. 1A show that the aqueous extract of I'm-Yunity was more potent in inhibiting cell proliferation than the comparable aqueous extract of I'm-Yunity-Too. Ethanolic extracts of the latter were slightly more active than the former in suppressing cell growth and, significantly more effective in inducing cell death (Fig. 1B).

I'm-Yunity and I'm-Yunity-Too extracts affect $N F-\kappa B / I \kappa B$ expression. To determine whether $\mathrm{NF}-\kappa \mathrm{B}$ plays a role on cell growth inhibition by extracts of I'm-Yunity or I'm-Yunity-Too, the expression of $\mathrm{NF}-\kappa \mathrm{B} / \mathrm{I} \kappa \mathrm{B}$ was assayed by western blot analysis. Results in Fig. 2 show that the ethanolic extracts of I'm-Yunity-Too had a potent suppressive effect on the expression of NF- $\kappa \mathrm{B}$ p 65 throughout the duration of the experiment, and similarly inhibited $\mathrm{I} \kappa \mathrm{B}$ levels for 48 - and $72 \mathrm{~h}$-exposed cells. The NF- $\mathrm{B}$ p 65 modulatory effects were only transiently 
A

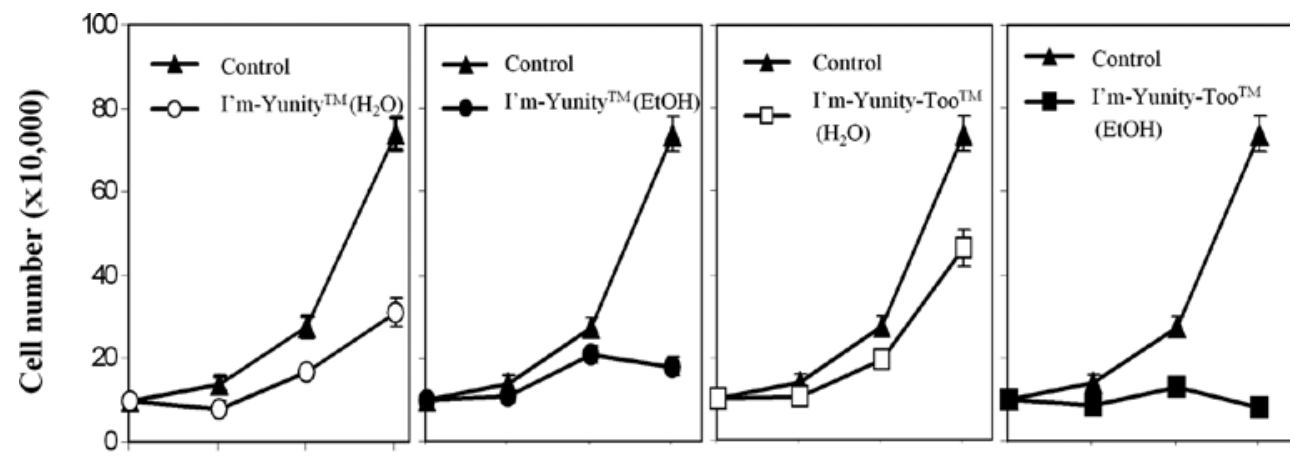

$\mathbf{B}$

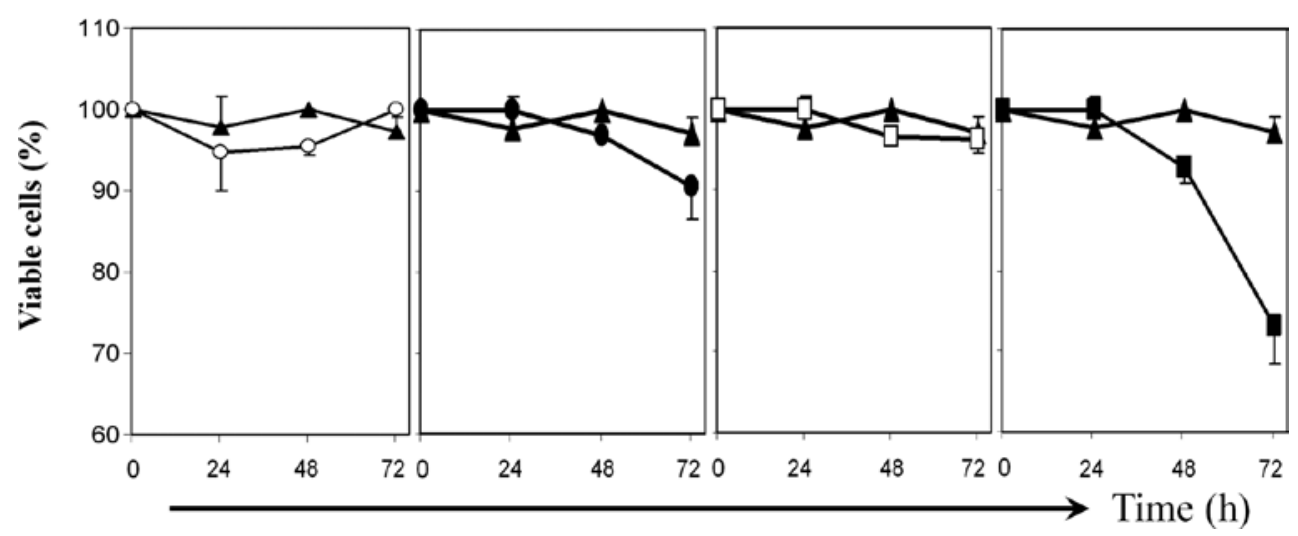

Figure 1. Effects of I'm-Yunity and I'm-Yunity-Too on HL-60 cell proliferation and viability. (A) Cells were treated with $10 \mu \mathrm{l} / \mathrm{ml}$ aqueous and ethanolic extracts of I'm-Yunity and I'm-Yunity-Too. Cell numbers were assayed at 24, 48 and $72 \mathrm{~h}$ post-treatment using a hemocytometer. (B) Cell viability following treatment was measured as described in Materials and methods. The results are presented as mean $\pm \mathrm{SD}$ of a minimum of three separate experiments.

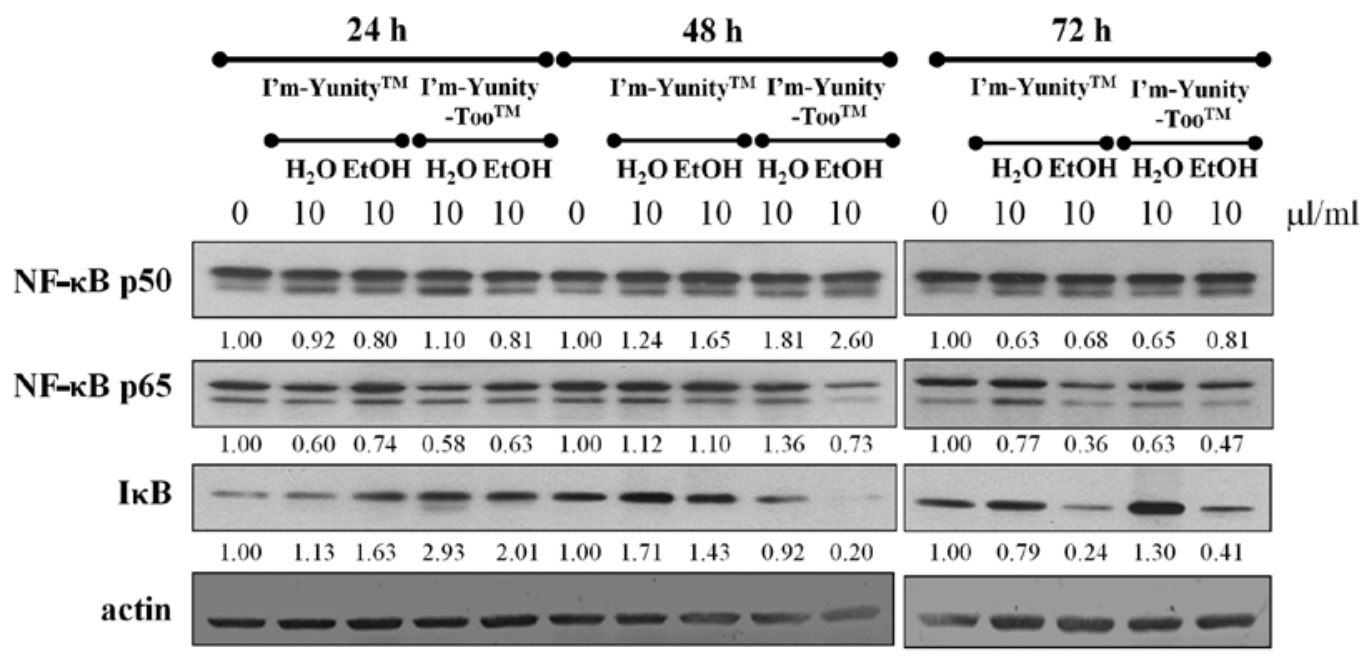

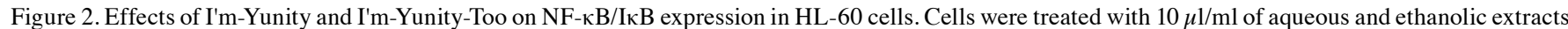
of I'm-Yunity and I'm-Yunity-Too for 24,48 and $72 \mathrm{~h}$. The cells were collected and total protein extracts were prepared as described in Materials and methods.

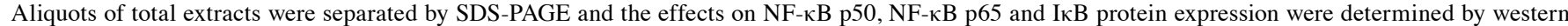
blot analysis, using actin as a loading control to quantify changes in the expression of the referenced genes. The intensity of the specific immunoreactive bands was quantified by densitometry and expressed as a fold difference against actin.

observed in cells treated for $24 \mathrm{~h}$ with aqueous extracts of I'm-Yunity or I'm-Yunity-Too, while for I'm-Yunity the ethanolic extract-exposed cell treatment for $72 \mathrm{~h}$ also significantly

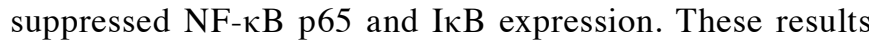
suggest that the ethanolic extract of I'm-Yunity-Too has a significantly more pronounced effect in reducing the level of the cell survival gene, $\mathrm{NF}-\mathrm{\kappa B}$, as compared to extracts derived from I'm-Yunity, suggesting that differential gene modulatory effects exist between the aqueous and ethanolic extracts of I'm-Yunity and I'm-Yunity-Too.

Cell cycle control by extracts of I'm-Yunity and I'm-Yunity-Too. To examine whether I'm-Yunity- and I'm-Yunity-Too-induced cell growth inhibition involves changes in the cell cycle phase transition, flow cytometry was performed in the control and exposed cells. Results in Fig. 3A show that HL-60 cells treated 
A

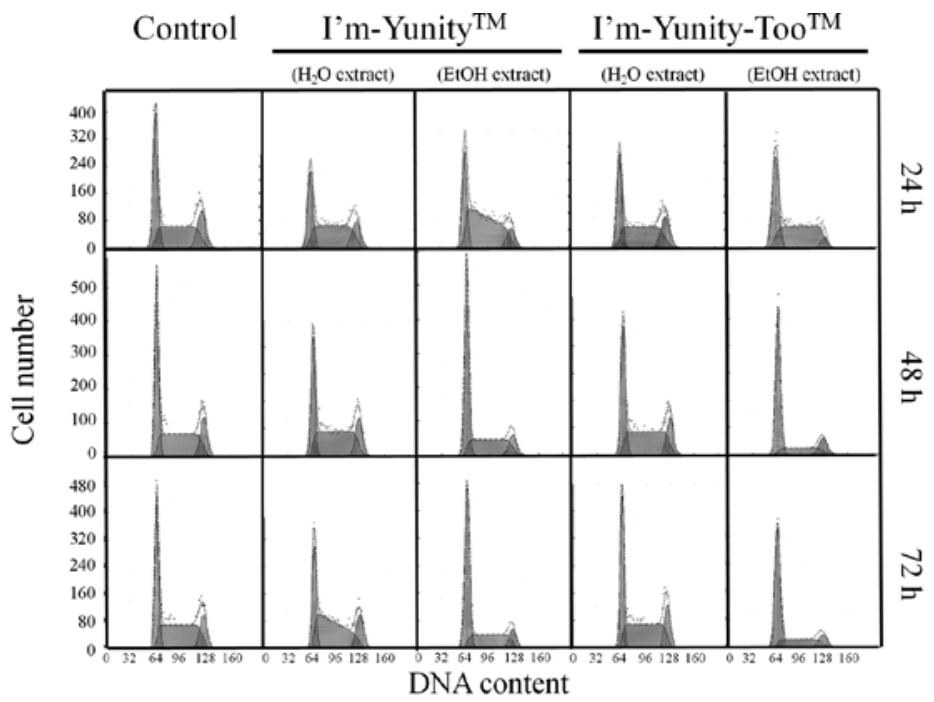

\begin{tabular}{|c|c|c|c|c|c|c|c|c|c|c|c|c|c|c|c|}
\hline \multirow[t]{2}{*}{$\mathrm{HL}-60$} & \multicolumn{3}{|c|}{ control } & \multicolumn{3}{|c|}{$\begin{array}{l}\text { I'm-Yunity' } \\
\left(\mathrm{H}_{2} \mathrm{O} \text { extract) }\right.\end{array}$} & \multicolumn{3}{|c|}{$\begin{array}{l}\text { I'm-Yunity }{ }^{\mathrm{TM}} \\
\text { (EtOH extract) }\end{array}$} & \multicolumn{3}{|c|}{$\begin{array}{c}\text { I'm-Yunity-Too }{ }^{\mathrm{TM}} \\
\left(\mathrm{H}_{2} \mathrm{O} \text { extract }\right)\end{array}$} & \multicolumn{3}{|c|}{$\begin{array}{l}\text { I'm-Yunity-Too }{ }^{\mathrm{TM}} \\
\text { (EtOH extract) }\end{array}$} \\
\hline & $\mathrm{G}_{1}$ & $\mathrm{~s}$ & $\mathrm{G}_{2} / \mathrm{M}$ & $\mathrm{G}_{1}$ & $\mathrm{~S}$ & $\mathrm{G}_{2} / \mathrm{M}$ & $\mathrm{G}_{1}$ & S & $\mathrm{G}_{2} / \mathrm{M}$ & $\mathrm{G}_{1}$ & S & $\mathrm{G}_{2} / \mathrm{M}$ & $\mathrm{G}_{1}$ & $\mathrm{~s}$ & $\mathrm{G}_{2} / \mathrm{M}$ \\
\hline $24 \mathrm{~h}$ & 37.7 & 44.5 & 17.8 & 29.2 & 55.5 & 15.3 & 26.1 & 64.7 & 9.2 & 31.8 & 50.4 & 17.7 & 39.9 & 55.7 & 4.4 \\
\hline $48 \mathrm{~h}$ & 38.7 & 46.0 & 15.2 & 30.4 & 53.7 & 15.9 & 54.5 & 35.9 & 9.6 & 34.1 & 51.2 & 14.8 & 66.4 & 21.5 & 12.1 \\
\hline $72 \mathrm{~h}$ & 35.0 & 50.5 & 14.5 & 26.3 & 57.1 & 16.6 & 56.5 & 33.6 & 9.9 & 34.1 & 50.5 & 15.3 & 61.2 & 28.1 & 10.7 \\
\hline
\end{tabular}

B

$24 \mathrm{~h}$

$48 \mathrm{~h}$

$72 \mathrm{~h}$

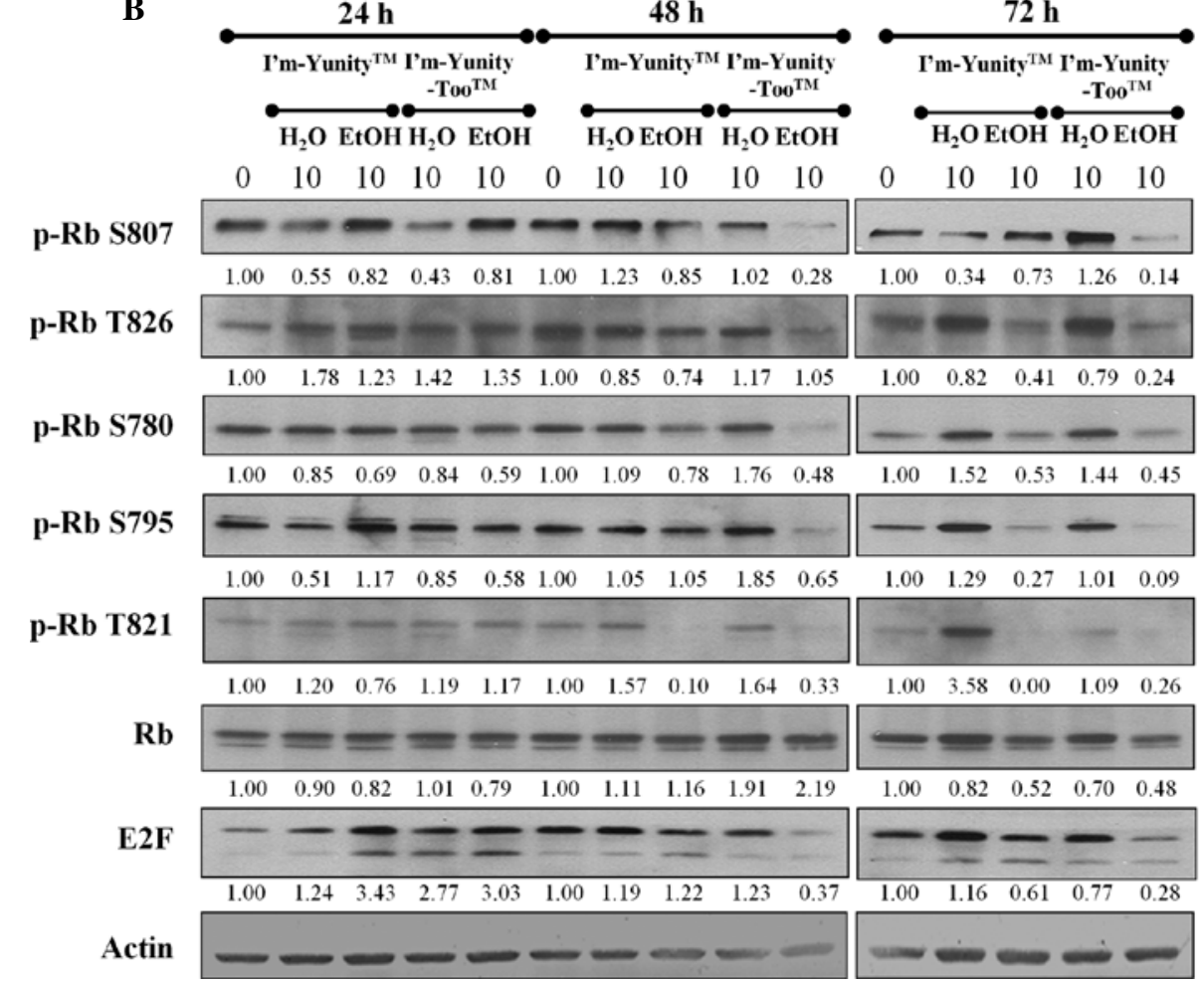

Figure 3. Effects of aqueous and ethanolic extracts of I'm-Yunity and I'm-Yunity-Too on the changes in cell cycle phase distribution and the expression of Rb, $\mathrm{Rb}$ phosphorylation and E2F in HL-60 cells. (A) At $24-72 \mathrm{~h}$ post-treatment with $10 \mu \mathrm{l} / \mathrm{ml} \mathrm{I}$ 'm-Yunity and I'm-Yunity-Too extracts, the cells were harvested and cell cycle analysis was performed. (B) Expression of total Rb, E2F and phosphorylated Rb at Ser780, Ser795, Ser807, Thr821 and Thr826 was determined by immunoblot analysis. Blots were quantified; relative expression was presented as the fold difference in comparison with the level of the loading control, actin.

with ethanolic extracts of I'm-Yunity-Too were restricted in the $\mathrm{G}_{1} \rightarrow \mathrm{S}$ progression. Fig. 3A also shows a decrease in cell fraction accumulated in the $\mathrm{G}_{2} \mathrm{M}$ phase of the cell cycle, as compared to the untreated cells. These effects were most notable in 48-72 h exposed cells. By contrast, in cells treated for $24 \mathrm{~h}$ by aqueous and ethanolic extracts of I'm-Yunity and
I'm-Yunity-Too, the primary cell cycle involved attenuation in the $\mathrm{S} \rightarrow \mathrm{G}_{2} \mathrm{M}$ phase transition.

To elucidate control of $\mathrm{G}_{1} \rightarrow \mathrm{S}$ in HL-60 cells treated with aqueous/ethanolic extracts of I'm-Yunity and I'm-Yunity-Too, changes in $\mathrm{Rb} / \mathrm{E} 2 \mathrm{~F}$ expression and the state of phosphorylation of $\mathrm{Rb}$ were determined. Western blot analysis demonstrated 
A

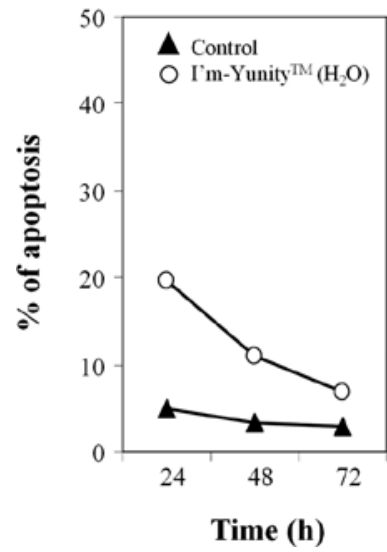

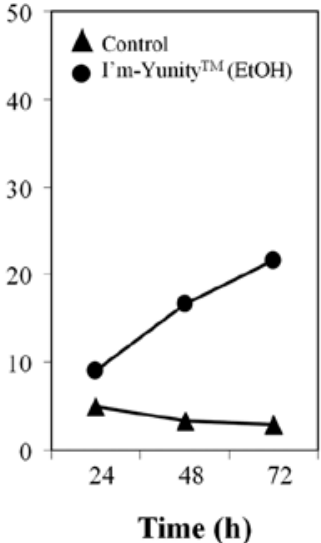

Time (h)
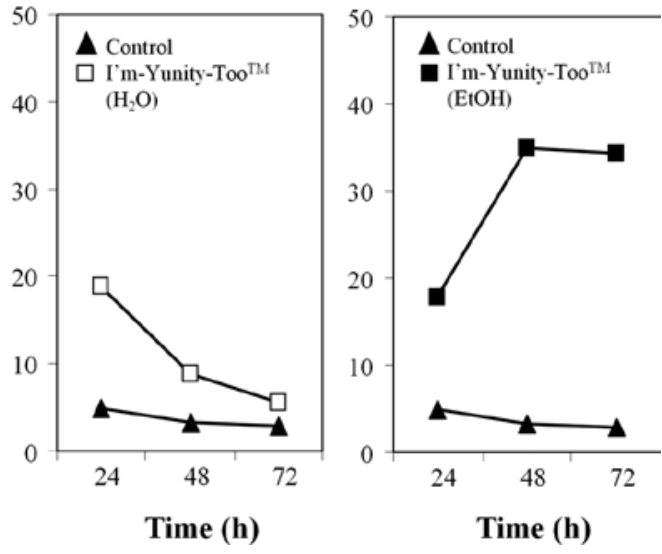

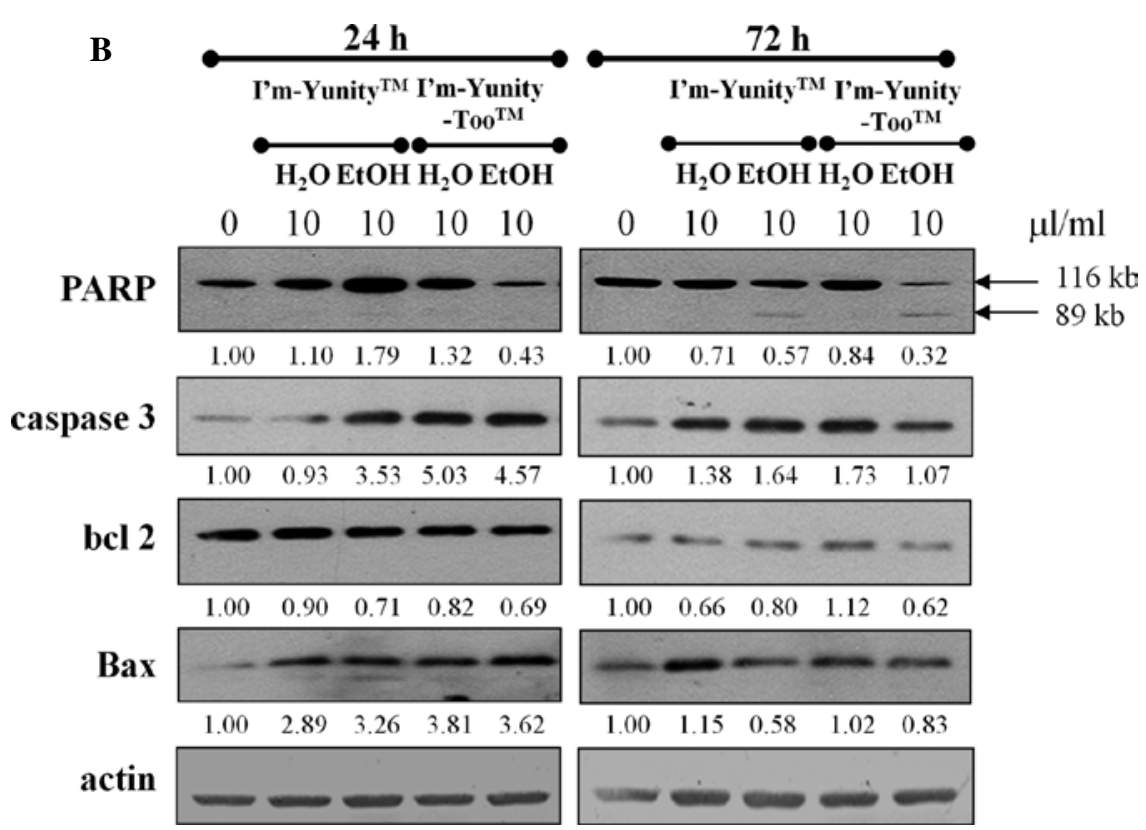

Figure 4. Effects of I'm-Yunity and I'm-Yunity-Too on the induction of apoptosis and expression of PARP, caspase 3, Bax and bcl 2. (A) HL-60 cells were treated with $10 \mu \mathrm{l} / \mathrm{ml}$ aqueous and ethanolic extracts of I'm-Yunity and I'm-Yunity-Too for 24-72 h. Induction of cell death by treatment was demonstrated by the appearance of the sub- $\mathrm{G}_{1}$ fraction in flow cytometric analysis. The extent of induction of apoptosis by treatment was calculated and presented as a percentage of the total cell population. (B) Following a 24- and 72-h treatment with $10 \mu \mathrm{l} / \mathrm{ml}$ I'm-Yunity and I'm-Yunity-Too extracts, cells were harvested and total protein extracts were prepared as described in Materials and methods. Aliquots of total extracts were separated by SDS-PAGE and western blot analysis was performed to reveal the cleavages of PARP from 112- to 89-kDa fragment. Expression of caspase 3, bcl 2 and bax was also determined. Blots were quantified and relative expression levels of the referenced proteins were shown as fold differences against the loading control, actin.

that while aqueous and ethanolic extracts of I'm-Yunity and I'm-Yunity-Too did not change the expression of total Rb, they markedly reduced the levels of $\mathrm{Rb}$ phosphorylation at Ser807, Ser780, Thr821, as well as E2F, in 48 h-exposed cells (Fig. 3B). Furthermore, the most pronounced changes occurred in cells treated with ethanolic extracts of I'm-Yunity-Too for 48 and $72 \mathrm{~h}$, reinforcing that extracts derived from the combination mushroom have biological activities that are different from the ethanolic extract prepared from the single mushroom I'm-Yunity. These findings also suggest that ethanolic extracts of I'm-Yunity and I'm-Yunity-Too exert multiple cell cycle effects in HL-60 cells manifested as specific checkpoint arrest.

Targeting the induction of apoptosis by I'm-Yunity and I'm-Yunity-Too. Results in Figs. 1B and 4A show that the ethanolic extract of I'm-Yunity-Too induced significantly more effective cell death as compared to the ethanolic extract of I'm-Yunity, leading to subsequent analysis of molecular determinants possibly contributing to such effects. Fig. 4B shows that treatment with aqueous and ethanolic extracts of I'm-Yunity and I'm-Yunity-Too at 24 and $72 \mathrm{~h}$ elevated the expression of caspase 3 as well as Bax, both integral to the induction of apoptosis. Corroborative evidence for apoptosis is derived from a reduction in the expression of PARP in cells treated for $72 \mathrm{~h}$ with ethanolic, but not aqueous, extracts of I'm-Yunity and I'm-Yunity-Too, concomitant with the increase in the cleaved $89-\mathrm{kDa}$ product from the $112-\mathrm{kDa}$ precursor.

\section{Discussion}

The adjunctive potential of Ganoderma lucidum in the management of cancer patients has been previously reported (51). However, the mechanisms by which it exerts efficacy have not been fully elucidated. It also remains to be determined whether, when administered in combination, the mechanisms generate novel activities. 
In the present study, HL-60 cells cultured in vitro were used to analyze the effects of I'm-Yunity (Coriolus versicolor) relative to I'm-Yunity-Too (Coriolus versicolor combined with Ganoderma lucidum) with regard to proliferation and induction of apoptosis. We demonstrated that I'm-Yunity and I'm-Yunity-Too significantly inhibited HL-60 proliferation, in concordance with the downregulation of NF- $\mathrm{B}$ p 65 expression. We also observed differences concerning changes in $\mathrm{I} \kappa \mathrm{B}$ expression between the aqueous and ethanolic extracts of I'm-Yunity and I'm-Yunity-Too. Thus, cells treated with ethanolic extracts for $72 \mathrm{~h}$ showed the downregulation of IкB, which was not affected in cells treated with aqueous extracts.

Studies directed at determining the mechanism controlling cell cycle progression by I'm-Yunity and I'm-Yunity-Too revealed ethanolic extracts derived from I'm-Yunity-Toorestricted cells in the $G_{1}$ compared to the untreated cells, the effects of which were most pronounced in cells treated for 48-72 $\mathrm{h}$ and occurred in coordination with profound loss of E2F and the downregulation of the Rb phosphorylation sites Ser780, Ser795, Thr821 and Thr826, respectively. In terms of induction of apoptosis, ethanolic extract of I'm-Yunity-Too caused more cell death, as compared to those of I'm-Yunity. We also found that ethanolic extract-treated cells induced more cell death at $72 \mathrm{~h}$ as compared to aqueous extract-treated cells, based on PARP cleavage which only appeared in ethanolic extract-treated cells.

Taken together, the results suggest that distinct differences exist regarding the cell and gene regulatory effects of aqueous and ethanolic extracts of I'm-Yunity-Too, as compared to comparable extracts derived from I'm-Yunity. In addition, extracts from the combination mushroom have different biological activity, compared to ethanolic extract from the single mushroom I'm-Yunity. Additional experiments and more detailed analysis are required to determine the underlying mechanisms involved.

\section{Acknowledgements}

Research in this report was supported in part by an unrestricted grant from the Hong Kong Healthcare Center, Ltd. We acknowledge with thanks the support of Ms. Vivien Chou and her family on mechanistic studies of medicinal mushrooms in the prevention and management of chronic diseases in humans.

\section{References}

1. Doll R and Peto R: The causes of cancer: quantitative estimates of avoidable risks of cancer in the United States today. J Natl Cancer Inst 66: 1191-1308, 1981

2. Steinmetz KA and Potter JD: Vegetables, fruit, and cancer. I. Epidemiology. Cancer Causes Control 2: 325-357, 1991.

3. Steinmetz KA and Potter JD: Vegetables, fruit, and cancer prevention: a review. J Am Diet Assoc 96: 1027-1039, 1996.

4. Willett WC: Diet, nutrition, and avoidable cancer. Environ Health Perspect 103 (Suppl 8): 165-170, 1995.

5. Link LB and Potter JD: Raw versus cooked vegetables and cancer risk. Cancer Epidemiol Biomarkers Prev 13: 1422-1435, 2004.

6. Potter JD: Vegetables, fruit, and cancer. Lancet 366: 527-530, 2005.

7. Surh YJ: Cancer chemoprevention with dietary phytochemicals. Nat Rev Cancer 3: 768-780, 2003.

8. Thomasset SC, Berry DP, Garcea G, Marczylo T, Steward WP and Gescher AJ: Dietary polyphenolic phytochemicals - promising cancer chemopreventive agents in humans? A review of their clinical properties. Int J Cancer 120: 451-458, 2007.
9. de Kok TM, van Breda SG and Manson MM: Mechanisms of combined action of different chemopreventive dietary compounds: a review. Eur J Nutr 47 (Suppl 2): 51-59, 2008.

10. Link A, Balaguer F and Goel A: Cancer chemoprevention by dietary polyphenols: promising role for epigenetics. Biochem Pharmacol 80: 1771-1792, 2010.

11. Lee KW, Bode AM and Dong Z: Molecular targets of phytochemicals for cancer prevention. Nat Rev Cancer 11: 211-218, 2011.

12. Sliva D: Ganoderma lucidum (Reishi) in cancer treatment. Integr Cancer Ther 2: 358-364, 2003.

13. Yuen JW and Gohel MD: Anticancer effects of Ganoderma lucidum: a review of scientific evidence. Nutr Cancer 53: 11-17, 2005.

14. Paterson RR: Ganoderma - a therapeutic fungal biofactory. Phytochemistry 67: 1985-2001, 2006.

15. Zhou X,Lin J, Yin Y,Zhao J, Sun X and Tang K: Ganodermataceae: natural products and their related pharmacological functions. Am J Chin Med 35: 559-574, 2007.

16. Zhong JJ and Xiao JH: Secondary metabolites from higher fungi: discovery, bioactivity, and bioproduction. Adv Biochem Eng Biotechnol 113: 79-150, 2009.

17. Zhang J, Tang Q, Zhou C, et al: GLIS, a bioactive proteoglycan fraction from Ganoderma lucidum, displays anti-tumour activity by increasing both humoral and cellular immune response. Life Sci 87: 628-637, 2010.

18. Wasser SP and Weis AL: Therapeutic effects of substances occurring in higher Basidiomycetes mushrooms: a modern perspective. Crit Rev Immunol 19: 65-96, 1999.

19. Cui J and Chisti Y: Polysaccharopeptides of Coriolus versicolor: physiological activity, uses, and production. Biotechnol Adv 21: 109-122, 2003.

20. Kidd PM: The use of mushroom glucans and proteoglycans in cancer treatment. Altern Med Rev 5: 4-27, 2000.

21. Ho CY, Kim CF, Leung KN, et al: Coriolus versicolor (Yunzhi) extract attenuates growth of human leukemia xenografts and induces apoptosis through the mitochondrial pathway. Oncol Rep 16: 609-616, 2006.

22. Nicandro JP, Tsourounis C, Frassetto L and Guglielmo BJ: In vivo effect of I'm-Yunity on hepatic cytochrome P450 3A4. J Herb Pharmacother 7: 39-56, 2007.

23. Hui KP, Sit WH and Wan JM: Induction of S phase cell arrest and caspase activation by polysaccharide peptide isolated from Coriolus versicolor enhanced the cell cycle-dependent activity and apoptotic cell death of doxorubicin and etoposide, but not cytarabine in HL-60 cells. Oncol Rep 14: 145-155, 2005.

24. Hsieh TC, Kunicki J, Darzynkiewicz Z and Wu JM: Effects of extracts of Coriolus versicolor (I'm-Yunity) on cell-cycle progression and expression of interleukins- 1 beta, -6 , and -8 in promyelocytic HL-60 leukemic cells and mitogenically stimulated and nonstimulated human lymphocytes. J Altern Complement Med 8: 591-602, 2002.

25. Zeng F, Hon CC, Sit WH, et al: Molecular characterization of Coriolus versicolor PSP-induced apoptosis in human promyelotic leukemic HL-60 cells using cDNA microarray. Int J Oncol 27: 513-523, 2005.

26. Hsieh TC, Wu P, Park S and Wu JM: Induction of cell cycle changes and modulation of apoptogenic/anti-apoptotic and extracellular signaling regulatory protein expression by water extracts of I'm-Yunity (PSP). BMC Complement Altern Med 6: 30, 2006.

27. Hsieh TC and Wu JM: Differential control of growth, cell cycle progression, and gene expression in human estrogen receptor positive MCF-7 breast cancer cells by extracts derived from polysaccharopeptide I'm-Yunity and Danshen and their combination. Int J Oncol 29: 1215-1222, 2006.

28. Hu H,Ahn NS, Yang X,Lee YS and Kang KS: Ganoderma lucidum extract induces cell cycle arrest and apoptosis in MCF-7 human breast cancer cell. Int J Cancer 102: 250-253, 2002.

29. Tsang KW, Lam CL, Yan C, et al: Coriolus versicolor polysaccharide peptide slows progression of advanced non-small cell lung cancer. Respir Med 97: 618-624, 2003.

30. Wong CK, Bao YX, Wong EL, Leung PC, Fung KP and Lam CW: Immunomodulatory activities of Yunzhi and Danshen in post-treatment breast cancer patients. Am J Chin Med 33: 381-395, 2005.

31. Wan JM, Sit WH and Louie JC: Polysaccharopeptide enhances the anticancer activity of doxorubicin and etoposide on human breast cancer cells ZR-75-30. Int J Oncol 32: 689-699, 2008.

32. Luk SU, Lee TK, Liu J, et al: Chemopreventive effect of PSP through targeting of prostate cancer stem cell-like population. PLoS One 6: e19804, 2011. 
33. Jiang J, Slivova V, Harvey K, Valachovicova T and Sliva D: Ganoderma lucidum suppresses growth of breast cancer cells through the inhibition of Akt/NF-kappaB signaling. Nutr Cancer 49: 209-216, 2004.

34. Yue GG, Fung KP, Tse GM, Leung PC and Lau CB: Comparative studies of various ganoderma species and their different parts with regard to their antitumor and immunomodulating activities in vitro. J Altern Complement Med 12: 777-789, 2006.

35. Jiang J, Grieb B, Thyagarajan A and Sliva D: Ganoderic acids suppress growth and invasive behavior of breast cancer cells by modulating AP-1 and NF- $\mathrm{BB}$ signaling. Int J Mol Med 21: $577-584,2008$

36. Weng CJ, Chau CF, Yen GC, Liao JW, Chen DH and Chen KD Inhibitory effects of Ganoderma lucidum on tumorigenesis and metastasis of human hepatoma cells in cells and animal models. J Agric Food Chem 57: 5049-5057, 2009.

37. Thyagarajan A, Jedinak A, Nguyen $\mathrm{H}$, et al: Triterpenes from Ganoderma lucidum induce autophagy in colon cancer through the inhibition of p38 mitogen-activated kinase (p38 MAPK). Nutr Cancer 62: 630-640, 2010.

38. Hsieh TC and Wu JM: Suppression of proliferation and oxidative stress by extracts of Ganoderma lucidum in the ovarian cancer cell line OVCAR-3. Int J Mol Med 28: 1065-1069, 2011.

39. Wu G, Qian Z, Guo J, et al: Ganoderma lucidum extract induces G1 cell cycle arrest, and apoptosis in human breast cancer cells Am J Chin Med 40: 631-642, 2012.

40. Bao PP, Lu W, Cui Y, et al: Ginseng and Ganoderma lucidum use after breast cancer diagnosis and quality of life: a report from the Shanghai Breast Cancer Survival Study. PLoS One 7: e39343, 2012 .

41. DiPietrantonio AM, Hsieh TC, Olson SC and Wu JM: Regulation of G1/S transition and induction of apoptosis in HL-60 leukemia cells by fenretinide (4HPR). Int J Cancer 78: 53-61, 1998

42. DiPietrantonio AM, Hsieh TC, Juan G, Traganos F, Darzynkiewicz Z and Wu JM: Fenretinide-induced caspase 3 activity involves increased protein stability in a mechanism distinct from reactive oxygen species elevation. Cancer Res 60 $4331-4335,2000$
43. Elangovan S and Hsieh TC: Control of cellular redox status and upregulation of quinone reductase NQO1 via Nrf2 activation by $\alpha$-lipoic acid in human leukemia HL-60 cells. Int J Oncol 33: 833-838, 2008

44. Bedner E, Burfeind P, Hsieh TC, et al: Cell cycle effects and induction of apoptosis caused by infection of HL-60 cells with human granulocytic ehrlichiosis pathogen measured by flow and laser scanning cytometry. Cytometry 33: 47-55, 1998.

45. Smolewski P, Bedner E, Du L, et al: Detection of caspases activation by fluorochrome-labeled inhibitors: multiparameter analysis by laser scanning cytometry. Cytometry 44: 73-82, 2001.

46. Hsieh TC, Aguero-Rosenfeld ME, Wu JM, et al: Cellular changes and induction of apoptosis in human promyelocytic HL-60 cells infected with the agent of human granulocytic ehrlichiosis (HGE). Biochem Biophys Res Commun 232: 298-303, 1997.

47. Hsieh TC, Yang CJ, Lin CY, Lee YS and Wu JM: Control of stability of cyclin D1 by quinone reductase 2 in CWR22Rv1 prostate cancer cells. Carcinogenesis 33: 670-677, 2012.

48. Yang CJ, Lin CY, Hsieh TC, Olson SC and Wu JM: Control of eotaxin-1 expression and release by resveratrol and its metabolites in culture human pulmonary artery endothelial cells. Am J Cardiovasc Dis 1: 16-30, 2011.

49. Hsieh TC: Uptake of resveratrol and role of resveratrol-targeting protein, quinone reductase 2 , in normally cultured human prostate cells. Asian J Androl 11: 653-661, 2009.

50. Hsieh TC: Antiproliferative effects of resveratrol and the mediating role of resveratrol targeting protein $\mathrm{NQO} 2$ in androgen receptor-positive, hormone-non-responsive CWR22Rv1 cells. Anticancer Res 29: 3011-3017, 2009.

51. Lin CN, Tome WP and Won SJ: Novel cytotoxic principles of Formosan Ganoderma lucidum. J Nat Prod 54: 998-1002, 1991. 